\title{
QUASI-HEREDITY OF ALGEBRAS AND THEIR FACTOR ALGEBRAS
}

\author{
CHANGCHANG XI
}

(Communicated by Maurice Auslander)

Dedicated to Professor Tiande Lei on the occasion of his 65th birthday

\begin{abstract}
Let $A$ be a finite-dimensional algebra over an algebraically closed field and denote by $N$ the Jacobson radical of $A$. If there is an integer $i \geq 2$ such that $A / N^{i}$ is quasi-hereditary, then $A$ is quasi-hereditary.
\end{abstract}

Let $A$ be a finite-dimensional algebra over an algebraically closed field $k$. By $N$ we denote the Jacobson radical of $A$. An ideal of $A$ is called a heredity ideal of $A$ if it satisfies (1) $J^{2}=J,(2) J N J=0$, and (3) $J$ is a projective left $A$-module. We recall that the algebra $A$ is said to be quasi-hereditary provided there is a chain

$$
0 \subset J_{1} \subset J_{2} \subset \cdots \subset J_{n}=A
$$

of ideals of $A$ such that $J_{i} / J_{i-1}$ is a heredity ideal of $A / J_{i-1}$ for all $i=$ $1, \ldots, n$. Some basic properties on quasi-hereditary algebras may be found in [DR]. The aim of this note is to show the following: If the algebra $A$ is not quasi-hereditary, then, for any $i \geq 2$, the factor algebra $A / N^{i}$ never becomes a quasi-hereditary algebra.

Throughout this note all algebras are finite-dimensional $k$-algebras with 1 , module means finitely generated left module. By $\bar{a}$ (or $\bar{J}$ ) we denote the image of $a \in A$ (or $J \subseteq A$ ) under the canonical map $A \rightarrow A / I$, where $I$ is an ideal of $A$.

The above-mentioned result may be reformulated as the following theorem.

Theorem 1. Let $A$ be a basic connected algebra with Jacobson radical $N$. If $A / N^{i}$ is quasi-hereditary for some $i \geq 2$, then $A$ is quasi-hereditary.

To prove this result we need some preparations.

Lemma 2. Let $A$ be a basic algebra and $e$ be a primitive idempotent such that $J=A e A$ is a heredity ideal of $A$. Then $e A e \cong k$.

Proof. Since the field $k$ is algebraically closed and the Jacobson radical of $e A e$ is $e N e$, it follows from the definition of a heredity ideal that $e N e=0$ and $e A e \cong k$.

Received by the editors September 18, 1990 and, in revised form, March 27, 1992.

1991 Mathematics Subject Classification. Primary 16P20, 16G10.

Key words and phrases. Heredity ideals, hereditary algebras. 
Lemma 3. Let $A$ be a basic connected algebra with radical $N$ such that $\bar{A}=$ $A / N^{i}$ is quasi-hereditary for some $i \geq 2$. Let $e$ be a primitive idempotent of $A$ such that $\bar{A} \bar{e} \bar{A}$ is a heredity ideal of $\bar{A}$. Then $J=A e A$ is a heredity ideal of $A$.

Proof. By [DR] an idempotent ideal $A e A$ of $A$ with $e N e=0$ is a heredity ideal of $A$ if and only if the multiplication map

$$
A e \otimes_{e A e} e A \rightarrow A e A
$$

is bijective. Since $\bar{A} \bar{e} \bar{A}$ is a heredity ideal of $\bar{A}$, the multiplication map

$$
\overline{N^{i-1} e} \otimes_{k} \overline{e N} \rightarrow \overline{N^{i-1} e N}=0
$$

is bijective. This implies that $\bar{N}^{i-1} \bar{e}=0$ or $\bar{e} \bar{N}=0$. Similarly, we consider the multiplication map

$$
\overline{N e} \otimes_{k} \overline{e N^{i-1}} \rightarrow \overline{N e N^{i-1}}=0 .
$$

This gives us that either $\overline{N e}=0$ or $\overline{e N^{i-1}}=0$. If $\overline{N e}=0$ or $\overline{e N}=0$, then we get $N e=0$ or $e N=0$. Thus $J=A e A$ is obviously a heredity ideal of $A$. Now let us assume $\overline{N e} \neq 0$ and $\overline{e N} \neq 0$. Then $\bar{N}^{i-1} \bar{e}=0$ and $\bar{e} \bar{N}^{i-1}=0$. It follows from $\overline{N^{i-1} e}=0$ that $N^{i-1} e=0$, since $N^{i-1} e \neq 0$ yields that $N N^{i-1} e$ is a proper submodule of $N^{i-1} e$. Similarly, there holds $e N^{i-1}=0$. In particular, we have $N^{i} e=0$ and $e N^{i}=0$, and therefore the canonical maps $A e \rightarrow \bar{A} \bar{e}, e A \rightarrow \bar{e} \bar{A}$ are bijective. On the one hand, it follows from $e N e \subseteq N^{i}$ that $e N e=0$. On the other hand, the canonical commutative diagram

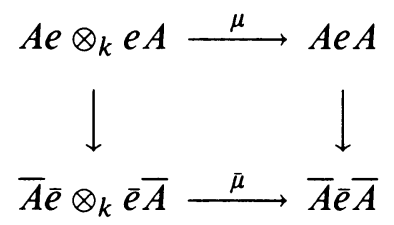

shows that with $\bar{\mu}$ also $\mu$ is injective. Hence $J=A e A$ is a heredity ideal of $A$.

The following lemma is an easy observation.

Lemma 4. Let $A$ be an artin algebra, $N=\operatorname{rad} A, \bar{A}=A / N^{i}$. Let $e$ be a primitive idempotent in $A$. Then $(A / A e A) / \operatorname{rad}^{i}(A / A e A) \cong \bar{A} / \overline{A e A}$.

Proof. Let $J=A e A$. Note that $\bar{J} \cong\left(J+N^{i}\right) / N^{i}$. From

$$
\begin{aligned}
\bar{A} / \bar{J} & \cong\left(A / N^{i}\right) /\left(\left(J+N^{i}\right) / N^{i}\right) \\
& \cong A /\left(J+N^{i}\right) \cong(A / J) /\left(\left(J+N^{i}\right) / J\right) \\
& \cong(A / J) /((J+N) / J)^{i}
\end{aligned}
$$

the lemma follows.

Proof of the theorem. We choose a complete set of pairwise nonisomorphic orthogonal primitive idempotents, say $e_{1}, \ldots, e_{n}$, such that for $J_{j}=$ $A\left(e_{1}+\cdots+e_{j}\right) A$, the chain

$$
0 \subset \bar{J}_{1} \subset \bar{J}_{2} \subset \cdots \subset \bar{J}_{n}=\bar{A}
$$


of ideals of $\bar{A}$ is a heredity chain for $\bar{A}$. Using Lemmas 3 and 4 repeatedly, we then get a heredity chain

$$
0 \subset J_{1} \subset J_{2} \subset \cdots \subset J_{n}=A
$$

of ideals of $A$. And therefore $A$ is quasi-hereditary.

Remarks 5. (1) The converse of the theorem is false. The following simple example is a desired one. Let $A$ be given by the quiver with the relation

$$
1 \circ \underset{\beta}{\stackrel{\alpha}{\rightleftarrows}} 02, \quad \alpha \beta=0 \text {. }
$$

Then it is easy to verify that $A$ is quasi-hereditary but $A / N^{2}$ is not.

(2) If one only assumes in the theorem that there exists an ideal $J \subset N^{2}$ such that $A / J$ is quasi-hereditary then $A$ may not be quasi-hereditary. Let $A$ be given by the above quiver with relations $\alpha \beta \alpha=0$ and $\beta \alpha \beta=0$. Then $A$ is not quasi-hereditary, but if one takes $J$ to be the socle of the projective module corresponding to the vertex 1 then $J \subset N^{2}$ and $A / J$ is isomorphic to the algebra displayed in (1), in particular, it is quasi-hereditary. Further examples may be found in [X].

\section{ACKNOWLEDGMENT}

I would like to thank Claus Michael Ringel for useful comments, Boris Hemkemeier for the help with typewriting, and the DFG for financial support.

\section{REFERENCES}

[DR] V. Dlab and C. M. Ringel, Quasi-hereditary algebras, Illinois J. Math. 33 (1989), 280-291.

[X] C. C. Xi, The structure of Schur algebras $S_{k}(n, p)$ for $n \geq p$, Canad. J. Math. 44 (1992), 665-672.

Fakultät für Mathematik, Universität Bielefeld, Postfach 8640, W-4800 Bielefeld 1, GERMANY

Current address: Department of Mathematics, Beijing Normal University, Beijing 100875, People's Republic of China 